\title{
Mean-square stability of a class of stochastic integral equations
}

\section{W.J. Padgett}

The object of this paper is to investigate under very general conditions the existence and mean-square stability of a random solution of a class of stochastic integral equations in the form

$$
x(t ; \omega)=h(t, x(t ; \omega))+\int_{0}^{t} K(t, s ; \omega) f(s, x(s ; \omega)) d s,
$$

for $t \geq 0$, where a random solution is a second order stochastic process $\{x(t ; \omega): t \geq 0\}$ which satisfies the equation almost certainly. A random solution $x(t ; \omega)$ is defined to be stable in mean-square if $E\left[|x(t ; \omega)|^{2}\right] \leq \rho$ for all $t \geq 0$ and some $\rho>0$ or exponentially stable in mean-square if $E\left[|x(t ; \omega)|^{2}\right] \leq \rho e^{-\alpha t}, t \geq 0$, for some constants $\rho>0$ and $\alpha>0$.

\section{Introduction}

Two important types of stochastic integral equations currently under study by mathematicians and probabilists are those involving $\hat{\text { tto }}$ or Ito-Doob type stochastic integrals and those involving mean-square integrals of second order stochastic processes. Stochastic integral equations involving the latter type of integrals occur in many probabilistic models for physical phenomena. For example, such equations arise in telephone traffic theory [5], [13], stochastic models for chemotherapy $[11],[12]$, stochastic theory of turbulence [14], systems 
theory [9], [15], [16], [18], and in many other situations [1], [2].

The purpose of this paper is to study the concept of stability in mean-square of random solutions of a certain class of stochastic integral equations involving second order stochastic processes. Specifically, we shall consider stochastic integral equations of the Hammerstein type in the form

$$
x(t ; \omega)=h(t, x(t ; \omega))+\int_{0}^{t} K(t, s ; \omega) f(s, x(s ; \omega)) d s,
$$

where $t \geq 0$ and

(i) $(\Omega, A, P)$ is a probability measure space, $P$ is a probability measure defined on the sigma-algebra $A$ of subsets of $\Omega$, and $\omega \in \Omega$;

(ii) $x(t ; \omega)$ is the unknown random variable for each $t \in R_{+}=[0, \infty)$;

(iii) $h(t, x)$ is a function defined for each $t \in R_{+}$and $x \in R$, the real numbers;

(iv) $K(t, 8 ; \omega)$ is the stochastic kernel defined for $0 \leq 8 \leq t<\infty$ and $\omega \in \Omega ;$ and

(v) $f(t, x)$ is a function of $t \in R_{+}$and $x \in R$.

Further conditions on the functions in equation (1.1) will be given in Section 2.

In [8], [17], and [19] equations similar to equation (1.1) were investigated. In this paper we shall study the existence, uniqueness, and mean-square stability properties of random solutions of (1.1) under more general conditions on the random functions $h, K$, and $f$ than those given in [8], [17], and [19]. The main results will be given in Theorems 3.1 and 3.2, and certain special cases of the main theorems will be presented in Section 4. Also, it should be noted that the stochastic Volterra integral equations in [17] and [19] may be obtained as special cases of (1.1) whenever $h(t, x(t ; \omega))$ is replaced by a function $h(t ; \omega)$ which does not depend on $x(t ; \omega)$. 
In order to obtain the objectives of this paper the concept of "admissibility" of Banach spaces [4] will be utilized.

\section{Preliminaries}

We shall make the following assumptions concerning the functions which appear in the stochastic integral equation (1.1):

(a) for each $t \in R_{+}, x(t ; \omega) \in L_{2}(\Omega, A, P)$, that is, $\left\{x(t ; \omega): t \in R_{+}\right\}$is a second order stochastic process on $R_{+},[7]$

(b) for each $t \in R_{+}, h(t, x(t ; \omega))$ and $f(t, x(t ; \omega))$ are in the space $L_{2}(\Omega, A, P)$;

(c) the mapping $(t, s) \rightarrow K(t, s ; \omega)$ is continuous from the set

$$
\Delta=\{(t, 8): 0 \leq s \leq t<\infty\}
$$

into the space $L_{\infty}(\Omega, A, P)$. Thus, for each $(t, s) \in \Delta$, we have

$$
K(t, s ; \omega) f(s, x(s ; \omega)) \in L_{2}(\Omega, A, P) .
$$

For each $(t, s) \in \Delta$ we shall denote the norm of $K(t, s ; \omega)$ in $L_{\infty}(\Omega, A, P)$ by

$$
\|K(t, 8 ; \omega)\|=P-\underset{\omega \epsilon \Omega}{\operatorname{ess} \sup }|K(t, 8 ; \omega)|
$$

and the norm of $x(t ; \omega)$ in $L_{2}(\Omega, A, P)$ for each $t \in R_{+}$by

$$
\begin{aligned}
\|x(t ; \omega)\|_{L_{2}(\Omega, A, P)} & =\left\{E\left[|x(t ; \omega)|^{2}\right]\right\}^{\frac{1}{2}} \\
& =\left\{\int_{\Omega}|x(t ; \omega)|^{2} d P(\omega)\right\}^{\frac{3}{2}} .
\end{aligned}
$$

DEFINITION 2.1. We define the space $C_{c}=C_{c}\left(R_{+}, L_{2}(\Omega, A, P)\right)$ to be the space of all continuous functions from $R_{+}$into the space $L_{2}(\Omega, A, P)$ with the topology of uniform convergence on every interval $[0, Q]$, $Q>0$. 
Note that $C_{c}$ is a locally convex space with the topology defined by the following sequence of seminorms:

$$
\|x(t ; \omega)\|_{n}=\sup _{0 \leq t \leq n}\|x(t ; \omega)\|_{L_{2}(\Omega, A, P)}, n=1,2, \ldots .
$$

DEFINITION 2.2. We shall denote by $C_{g}=C_{g}\left(R_{+}, L_{2}(\Omega, A, P)\right)$ the space of all continuous functions from $R_{+}$into $L_{2}(\Omega, A, P)$ such that there is a positive constant $\Gamma$ and a positive-valued continuous function $g$ on $R_{+}$satisfying

$$
\|x(t ; \omega)\|_{L_{2}(\Omega, A, P)} \leq \Gamma g(t), \quad t \in R_{+} .
$$

The norm in $C_{g}\left(R_{+}, L_{2}(\Omega, A, P)\right)$ is defined by

$$
\|x(t ; \omega)\|_{C_{g}}=\sup _{t \in R_{+}}\left\{\frac{\|x(t ; \omega)\|_{L_{2}(\Omega, A, P)}}{g(t)}\right\} .
$$

When the function $g(t)=1$ for all $t \in R_{+}$in Definition 2.2, we obtain the space of all bounded continuous functions from $R_{+}$into $L_{2}(\Omega, A, P)$, which will be denoted by $C=C\left(R_{+}, L_{2}(\Omega, A, P)\right)$. The space $C$ is the space of all second order stochastic processes defined on $R_{+}$ which are bounded in mean-square and continuous in mean-square.

Throughout this paper $B$ and $D$ will denote Banach spaces such that $B, D \subset C_{c}\left(R_{+}, L_{2}(\Omega, A, Z)\right)$.

DEFINITION 2.3. A pair of Banach spaces $(B, D)$ is said to be admissible with respect to a linear operator $T$ if $T(B) \subset D$.

A lemra in [17] states that if the spaces $B$ and $D$ have stronger topologies than the space $C_{C}\left(R_{+}, L_{2}(\Omega, A, P)\right)$ and the pair $(B, D)$ is admissible with respect to a continuous linear operator $T: C_{c} \rightarrow C_{c}$, then $T$ is continuous from $B$ into $D$. Thus, $T$ is bounded and for each $x(t ; \omega) \in B$, there exists a constant $M>0$ such that

$$
\|(T x)(t ; \omega)\|_{B} \leq M\|x(t ; \omega)\|_{D} .
$$


By a random solution of equation (I.I) we will mean a second order stochastic process on $R_{+}$which satisfies the equation $P$ almost everywhere.

In order to study the existence of a random solution of equation (1.1) we will employ the following fixed-point theorem, which was proven in [10]. Recall that an operator $T$ on a Banach space $B$ into itself is a nonlinear contraction if for all $x, y \in B$,

$$
\|T x-T y\|_{B} \leq \phi\left(\|x-y\|_{B}\right) \text {, }
$$

where $\phi$ is a real-valued continuous function satisfying $\phi(s)<s$ for $s>0$.

THEOREM 2.1 (Nashed and Wong [10]). Let $S$ be a closed, bounded convex subset of a Banach space and let $U$ and $V$ be operators on $S$ satisfying:

(i) $U(x)+V(y) \in S$ for alz $x, y \in S$;

(ii) $U$ is a completely continuous operator on $S$;

(iii) $V$ is a nonzinear contraction on $S$.

Then there exists a point $x^{*} \in S$ such that $U\left(x^{*}\right)+V\left(x^{*}\right)=x^{*}$.

Also, a fixed-point theorem of Boyd and Wong [3] which is a special case of Theorem 2.1 will be utilized to obtain the existence of a unique random solution of equation (1.1).

THEOREM 2.2 (Boyd and Wong [3]). If $V$ is a nonlinear contraction on a Bconach space $B$, then $V$ has a unique fixed point in $B$.

Finally, we shall make the following definitions concerning the asymptotic behavior of a random solution of (1.1).

DEFINITION 2.4. A random solution of equation (1.1) is said to be

(a) stable in mean-square if there exists a positive constant $\rho$ such that $E\left[|x(t ; \omega)|^{2}\right] \leq \rho$ for all $t \in R_{+}$, or

(b) exponentially stable in mean-square if there exist positive constants $\rho$ and $\alpha$ such that $E\left[|x(t ; \omega)|^{2}\right] \leq \rho e^{-\alpha t}$ for all $t \in R_{+}$. 


\section{Existence of a random solution}

We shall now prove two general theorems concerning the existence of a random solution of equation (1.1).

THEOREM 3.1. Consider the stochastic integral equation (1.1) subject to the following conditions:

(i) $B$ and $D$ are Banach spaces stronger than $C_{c}\left(R_{+}, L_{2}(\Omega, A, P)\right)$ and $(B, D)$ is admissible with respect to the integral operator

$$
(T x)(t ; \omega)=\int_{0}^{t} K(t, s ; \omega) x(s ; \omega) d s, t \geq 0,
$$

where $K$ behaves as described in Section 2;

(ii) $x(t ; \omega)+f(t, x(t ; \omega))$ is an operator from

$$
S=\left\{x(t ; \omega) \in D:\|x(t ; \omega)\|_{D} \leq \rho\right\}
$$

into $B$ satisfying

$$
\|f(t, x(t ; \omega))-f(t, y(t ; \omega))\|_{B} \leq \phi\left(\|x(t ; \omega)-y(t ; \omega)\|_{D}\right),
$$

where $\rho>0$ is a constant and $\phi$ is a real-valued continuous function such that $\phi(s)<s$ for $s>0$;

(iii) $x(t ; \omega) \rightarrow h(t, x(t ; \omega))$ is a completely continuous operator from $S$ into itself such that for some $\gamma>0$, $\|h(t, x(t ; \omega))\|_{D} \leq \gamma$.

Then there exists in $S$ a random solution of equation (1.1), provided $M_{T}<1$ and

$$
\gamma+M_{T}\|f(t, 0)\|_{B} \leq \rho\left(1-M_{T}\right),
$$

where $M_{T}$ is the norm of $T$.

Proof. Define the operator $V$ from $S$ into $D$ by

$$
(V x)(t ; \omega)=\int_{0}^{t} K(t, s ; \omega) f(s, x(s ; \omega)) d s .
$$

Let $x(t ; \omega), y(t ; \omega) \in S$. Then 


$$
\|h(t, x(t ; \omega))+(V y)(t ; \omega)\|_{D} \leq\|h(t, x(t ; \omega))\|_{D}+M_{T}\|f(t, y(t ; \omega))\|_{B}
$$

by condition (i) of the theorem and the remark following Definition 2.3. But, since

$$
\|f(t, y(t ; \omega))\|_{B} \leq \phi\left(\|y(t ; \omega)-0\|_{D}\right)+\|f(t, 0)\|_{B}
$$

by condition (ii), we have from condition (iv) that

$$
\begin{aligned}
\|h(t, x(t ; \omega))+(V y)(t ; \omega)\|_{D} \leq & \|h(t, x(t ; \omega))\|_{D} \\
& +M_{T} \phi\left(\|y(t ; \omega)\|_{D}\right)+M_{T}\|f(t, 0)\|_{B} \\
& \leq \gamma+M_{T}\|f(t, 0)\|_{B}+M_{T} \phi\left(\|y(t ; \omega)\|_{D}\right) .
\end{aligned}
$$

Now, from condition (ii) and the last hypothesis of the theorem, we obtain from inequality (3.2),

$$
\begin{aligned}
\|h(t, x(t ; \omega))+(V y)(t ; \omega)\|_{D} & <\rho\left(1-M_{T}\right)+M_{T}\|y(t ; \omega)\|_{D} \\
& \leq \rho\left(1-M_{T}\right)+M_{T^{\rho}} \\
& =\rho .
\end{aligned}
$$

That is, $h(t, x(t ; \omega))+(V y)(t ; \omega) \in S$ and condition $(i)$ of Theorem 2.I is satisfied.

Furthermore,

$$
\begin{aligned}
\|(V x)(t ; \omega)-(V y)(t ; \omega)\|_{D} & =\left\|\int_{0}^{t} K(t, s ; \omega)[f(s, x(s ; \omega))-f(s, y(s ; \omega))] d s\right\|_{D} \\
& \leq M_{T}\|f(t, x(t ; \omega))-f(t, y(t ; \omega))\|_{B} \\
& \leq \phi\left(\|x(t ; \omega)-y(t ; \omega)\|_{D}\right)
\end{aligned}
$$

by condition $(i i)$ and the hypothesis that $M_{T}<1$. Thus, $V$ is a nonlinear contraction operator on $S$.

Therefore, by condition (iii) and Theorem 2.1 there exists in $S$ a random solution of (1.1).

By imposing a slightly stronger condition on the stochastic process $h(t, x(t ; \omega))$, we can obtain the existence of a vonique random solution of (1.1).

THEOREM 3.2. Suppose the stochastic integral equation (1.1) 
satisfies the following conditions:

(i) scome as condition (i) of theorem 3.1;

(ii) same as condition (ii) of Theorem 3.1;

(iii) $x(t ; \omega) \rightarrow h(t, x(t ; \omega))$ is an operator from the set $S$ into $D$ satisfying

$$
\begin{aligned}
& \|h(t, x(t ; \omega))-h(t, y(t ; \omega))\|_{D} \leq \gamma \phi\left(\|x(t ; \omega)-y(t ; \omega)\|_{D}\right), \\
& \text { where } \gamma>0 \text { is a constant. }
\end{aligned}
$$

Then there exists in $S$ a unique random solution of equation (1.1), provided that $\gamma+M_{T}<1$ and

$$
\|h(t, 0)\|_{D}+M_{T}\|f(t, 0)\|_{B} \leq \rho\left(1-\gamma-M_{T}\right),
$$

where $M_{T}$ is the norm of the operator $T$.

Proof. Define the operator $V$ from $S$ into $D$ by

$$
(V x)(t ; \omega)=h(t, x(t ; \omega))+\int_{0}^{t} K(t, s ; \omega) f(s, x(s ; \omega)) d s .
$$

Let $x(t ; \omega) \in S$. We must show that $V$ is a nonlinear contraction on $S$ into itself. By conditions (i) and (ii) of the theorem

$$
\text { (3.3) } \begin{aligned}
\|(V x)(t ; \omega)\|_{D} & \leq\|h(t, x(t ; \omega))\|_{D}+\left\|\int_{0}^{t} K(t, s ; \omega) f(s, x(s ; \omega)) d s\right\|_{D} \\
& \leq\|h(t, x(t ; \omega))\|_{D}+M_{T}\|f(t, x(t ; \omega))\|_{B} .
\end{aligned}
$$

But since

$$
\|h(t, x(t ; \omega))\|_{D} \leq\|h(t, x(t ; \omega))-h(t, 0)\|_{D}+\|h(t, 0)\|_{D}
$$

and a similar inequality holds for $f(t, x(t ; \omega))$, we have from inequality (3.3) and conditions (ii) and (iii) that

$$
\begin{aligned}
\|(V x)(t ; \omega)\|_{D} & \leq \gamma \phi\left(\|x(t ; \omega)\|_{D}\right)+\|h(t, 0)\|_{D} \\
& \quad+M_{T} \phi\left(\|x(t ; \omega)\|_{D}\right)+M_{T}\|f(t, 0)\|_{B} \\
& <\left(\gamma+M_{T}\right)\|x(t ; \omega)\|_{D}+\|h(t, 0)\|_{D}+M_{T}\|f(t, 0)\|_{B} \\
& \leq \rho\left(\gamma+M_{T}\right)+\rho\left(1-\gamma-M_{T}\right)=\rho
\end{aligned}
$$


by the last hypothesis of the theorem. Hence, $V(S) \subset S$.

Now, let $x(t ; \omega), y(t ; \omega) \in S$. Then applying the hypotheses of the theorem, we have

$$
\begin{aligned}
& \|(V x)(t ; \omega)-(V y)(t ; \omega)\|_{D} \\
& \leq\|h(t, x(t ; \omega))-h(t, y(t ; \omega))\|_{D} \\
& \quad \quad \quad\left\|\int_{0}^{t} K(t, s ; \omega)[f(s, x(s ; \omega))-f(s, y(s ; \omega))] d s\right\|_{D} \\
& \leq\|h(t, x(t ; \omega))-h(t, y(t ; \omega))\|_{D}+M_{T}\|f(t, x(t ; \omega))-f(t, y(t ; \omega))\|_{B} \\
& \leq \gamma \phi\left(\|x(t ; \omega)-y(t ; \omega)\|_{D}\right)+M_{T} \phi\left(\|x(t ; \omega)-y(t ; \omega)\|_{D}\right) \\
& \leq \phi\left(\|x(t ; \omega)-y(t ; \omega)\|_{D}\right)
\end{aligned}
$$

since $\gamma+M_{T}<1$. Thus, $V$ is a nonlinear contraction on $S$ into itself, and by Theorem 2.2 there exists a unique random solution of equation $(1.1), x^{*}(t ; \omega) \in S$.

\section{Mean-square stability}

By choosing the Banach spaces $B$ and $D$ to be the spaces $C\left(R_{+}, L_{2}(\Omega, A, P)\right)$ or $C_{g}\left(R_{+}, L_{2}(\Omega, A, P)\right)$, we may obtain certain usefu工 special cases of Theorems 3.1 and 3.2 concerning the existence and meansquare stability of random solutions of (1.1).

Recall that an operator $h$ on the bounded set $S$ is completely continuous if the image $h(S)$ is relatively compact, that is, if every bounded sequence in $h(S)$ has a convergent subsequence.

Let $x(t ; \omega) \rightarrow h(t, x(t ; \omega))$ be an operator from the set

$$
S=\left\{x(t ; \omega) \in C:\|x(t ; \omega)\|_{C} \leq \rho\right\}
$$

into itself, where $\rho>0$ is a constant. From [6] it follows that $h(s) \subset C$ is relatively compact if $h$ is such that

$$
\text { (A) } \quad \lim _{\delta \rightarrow 0^{+}} \sup _{x \in S}\left\{\sup _{\substack{s, t \geq 0 \\|s-t|<\delta}}\left[\|h(t, x(t ; \omega))-h(s, x(s ; \omega))\|_{L_{2}(\Omega, A, P)}\right]\right\}=0
$$


and

(B) for each fixed $t \in R_{+}$every sequence in $\{h(t, x(t ; \omega)): x(t ; \omega) \in S\} \subset L_{2}(\Omega, A, P)$ has 8 convergent subsequence.

We shall now prove the following special case of Theorem 3.1.

THEOREM 4.1. Consider the stochastic integral equation (1.1) under the following conditions:

(i) there exist a positive number $\Gamma$ and a positive continuous function $g(t)$ on $R_{+}$such that

$$
\int_{0}^{t}\|K(t, s ; \omega)\| g(s) d s \leq \Gamma, \quad t \in R_{+} ;
$$

(ii) $x(t ; \omega) \rightarrow f(t, x(t ; \omega))$ is an operator from the set $S$ given by (4.1) into $C_{g}\left(R_{+}, L_{2}(\Omega, A, P)\right)$ satisfying for every $t \in R_{+}$,

$$
|f(t, x(t ; \omega))-f(t, y(t ; \omega))| \leq g(t) \phi\left(\|x(t ; \omega)-y(t ; \omega)\|_{C}\right)
$$

with probability one for $x(t ; \omega), y(t ; \omega) \in S$, where $\phi$ is a real-valued continuous function such that $\phi(s)<s$ for $s>0$;

(iii) $x(t ; \omega) \rightarrow h(t, x(t ; \omega))$ is an operator from $s$ into $C$ such that $|h(t, x(t ; \omega))| \leq \gamma$ with probability one for every $t \in R_{+}$, some $Y>0$ and such that (A) and (B) above are satisfied.

Then there exists a random solution $x^{*}(t ; \omega)$ of $(1.1)$ which is stable in mean-square, provided $\sup _{t \in R_{+}}|f(t, 0)| / g(t), \Gamma$ and $\gamma$ are sufficiently smazl.

Proof. By condition $(i)$ the pair of Banach spaces $\left(C_{g}, C\right)$ is admissible with respect to the integral operator 


$$
(T x)(t ; \omega)=\int_{0}^{t} K(t, 8 ; \omega) x(8 ; \omega) d s,
$$

since for $x(t ; \omega)^{\circ} \in C_{g}$,

$$
\begin{aligned}
\|(T x)(t ; \omega)\|_{L_{2}(\Omega, A, P)} & \leq \int_{0}^{t}\|K(t, s ; \omega)\| \cdot\|x(s ; \omega)\|_{L_{2}(\Omega, A, P)} d \theta \\
& \leq \sup _{t \in R_{+}}\left\{\frac{\|x(t ; \omega)\|_{L_{2}(\Omega, A, P)}}{g(t)}\right\} \int_{0}^{t}\|K(t, s ; \omega)\| g(s) d s \\
& \leq\|x(t ; \omega)\|_{C_{g} \cdot \Gamma}
\end{aligned}
$$

by definition of the norm in $C_{g}\left(R_{+}, L_{2}(\Omega, A, P)\right)$. That is, $(T x)(t ; \omega) \in C$.

It clearly follows from condition (ii) that for $x(t ; \omega), y(t ; \omega) \in S$,

$$
\|f(t, x(t ; \omega))-f(t, y(t ; \omega))\|_{C_{g}} \leq \phi\left(\|x(t ; \omega)-y(t ; \omega)\|_{C}\right) \text {, }
$$

and from condition (iii) that $h$ is completely continuous from $S$ into itself provided $\gamma$ is small enough.

Hence, by Theorem 3.1 there exists a random solution $x^{\star}(t ; \omega) \in C$ of equation (1.1) satis fying

$$
E\left[\left|x^{*}(t ; \omega)\right|^{2}\right] \leq \rho^{2}, t \in R_{+},
$$

whenever $\Gamma, \gamma$ and $\sup _{t \in R_{+}}|f(t, 0)| / g(t)$ are small enough.

Note that by Jensen's inequality $x^{*}(t ; \omega)$ is also stable in the mean

$$
E\left[\left|x^{*}(t ; \omega)\right|\right] \leq \rho, \quad t \in R_{+} \text {. }
$$

COROLLARY 4.2. Consider the stochastic integral equation (1.1) subject to the following conditions:

(i) $\int_{0}^{t}\|\| K(t, s ; \omega) \| d s \leq \Lambda, t \in R_{+}$, where $\Lambda$ is same positive constant;

(ii) $x(t, \omega) \rightarrow f(t, x(t ; \omega))$ is an operator from the set $s$ given 
by (4.1) into $C\left(R_{+}, L_{2}(\Omega, A, P)\right)$ such that for each $t \in R_{+}$, $|f(t, x(t ; \omega))-f(t, y(t ; \omega))| \leq \phi\left(\|x(t ; \omega)-y(\tau ; \omega)\|_{C}\right)$ with probability one for $x(t ; \omega), y(t ; \omega) \in S$, where $\phi$ is as given in Theorem 4.1 (ii);

(iii) some as condition (iii) of Theorem 4.1.

Then there exists a random solution $x^{\star}(t ; \omega) \in C$ of $(1.1)$ which is stable in mean-square, provided $\|f(t, 0)\|_{C}, \Lambda$ and $Y$ are small enough.

Proof. Take $g(t)=1$ for all $t \in R_{+}$in Theorem 4.1.

The following special case of Theorem 3.2 guarantees the existence of a unique random solution of (1.1) which is exponentially stable in meansquare.

THEOREM 4.3. Assume that the stochastic integral equation (1.1) satisfies:

(i) $\|K(t, s ; \omega)\| \leq \Gamma \exp [-\alpha(t-s)], 0 \leq s \leq t<\infty$, where $\Gamma>0$ and $\alpha>0$ are constants;

(ii) $x(t ; \omega) \rightarrow f(t, x(t ; \omega))$ is an operator on

$$
s_{g}=\left\{x(t ; \omega) \in C_{g}:\|x(t ; \omega)\|_{C_{g}} \leq \rho\right\}
$$

with values in $C_{g}\left(R_{+}, L_{2}(\Omega, A, P)\right)$ satisfying for each $t \in R_{+}$,

(4.3) $|f(t, x(t ; \omega))-f(t, y(t ; \omega))| \leq e^{-\beta t_{\phi}}\left(\|x(t ; \omega)-y(t ; \omega)\|_{C}\right)$

with probability one for $x(t ; \omega), y(t ; \omega) \in S_{g}$, where $0<\beta<\alpha, \rho>0$ is a constant, $\phi$ is as given in Theorem (4.I) (ii), and $g(t)=e^{-\beta t}, t \geq 0$;

(iii) $x(t ; \omega) \rightarrow h(t, x(t ; \omega))$ is an operator on $s_{g}$ with values in $C_{g}\left(R_{+}, L_{2}(\Omega, A, P)\right)$ satisfying for each $t \in R_{+}$, 


$$
|h(t, x(t ; \omega))-h(t, y(t ; \omega))| \leq e^{-\beta t} \gamma \phi\left(\|x(t ; \omega)-y(t ; \omega)\|_{C_{g}}\right)
$$

with probability one for $x(t ; \omega), y(t ; \omega) \in S_{g}$, where $\gamma>0$ is a constont.

Then there exists a unique random solution of equation (1.1) which is exponentially stable in mean-square, provided that. $r, \Gamma$ and $\|f(t, 0)\|_{C_{g}}$ are sufficiently small.

Proof. It is clear from condition (ii) that if inequality (4.3) is divided by $e^{-\beta t}$, we obtain for $x(t ; \omega), y(t ; \omega) \in S_{g}$,

$$
\sup _{t \in R_{+}}\left\{\frac{\|f(t, x(t ; \omega))-f(t, y(t ; \omega))\|_{L_{2}(\Omega, A, P)}}{e^{-\beta t}}\right\} \leq \phi\left(\|x(t ; \omega)-y(t ; \omega)\|_{C_{g}}\right)
$$

or

$$
\|f(t, x(t ; \omega))-f(t, y(t ; \omega))\|_{C_{g}} \leq \phi\left(\|x(t ; \omega)-y(t ; \omega)\|_{C_{g}}\right)
$$

when $g(t)=e^{-\beta t}, t \in R_{+}$. Similarly, from condition (iii) with $g(t)=e^{-\beta t}$, we have

$$
\|h(t, x(t ; \omega))-h(t, y(t ; \omega))\|_{C_{g}} \leq \gamma \phi\left(\|x(t ; \omega)-y(t ; \omega)\|_{C_{g}}\right) .
$$

We need only to show that condition $(i)$ implies that $\left(c_{g}, c_{g}\right)$ is admissible with respect to the linear operator $T$ given by equation (4.2). Let $x(t ; \omega) \in C_{g}\left(R_{+}, L_{2}(\Omega, A, P)\right)$ with $g(t)=e^{-B t}, t \geq 0$. Then from condition (i), 


$$
\begin{aligned}
\|(T x)(t ; \omega)\|_{L_{2}(\Omega, A, P)} & \leq \int_{0}^{t}\|K(t, s ; \omega)\| \cdot\|x(s ; \omega)\|_{L_{2}(\Omega, A, P)} d s \\
& \leq \Gamma e^{-\alpha t} \int_{0}^{t} e^{\alpha s} \frac{\|x(s ; \omega)\|_{L_{2}(\Omega, A, P)}}{e^{-\beta s}} e^{-\beta s} d s \\
& \leq \Gamma e^{-\alpha t} \sup _{t \in R_{+}}\left\{\frac{\|x(t ; \omega)\|_{L_{2}(\Omega, A, P)}}{e^{-\beta t}}\right\} \int_{0}^{t} e^{(\alpha-\beta) s} d s \\
& \leq \Gamma(\alpha-\beta)^{-1}\left\|_{x}(t ; \omega)\right\|_{C_{g}} e^{-\beta t},
\end{aligned}
$$

which means that $(T x)(t ; \omega) \in C_{g}\left(R_{+}, L_{2}(\Omega, A, P)\right)$.

Therefore, if $\Gamma, \gamma$ and $\|f(t, 0)\|_{C_{g}}$ are sufficiently small, by Theorem 3.2 there exists a unique random solution of (1.1), $x^{*}(t ; \omega) \in S_{g}$. That is,

$$
E\left[\left|x^{*}(t ; \omega)\right|^{2}\right] \leq \rho^{2} e^{-2 \beta t}, \quad t \in R_{+},
$$

which means that $x^{*}(t ; \omega)$ is exponentially stable in mean-square.

\section{References}

[1] A.T. Bharucha-Reld, "On the theory of rendom equations", Proc. Sympos. Appz. Math. 16, 40-69 (American Mathematical Society, Providence, Rhode Island, 1964).

[2] A.T. Bharucha-Reid, Rondom integral equations (Academic Press, New York, to be published).

[3] D.W. Boyd and J.S.W. Wong, "On nonlinear contractions", Proc. Amer. Math. Soc. 20 (1969), 458-464.

[4] C. Corduneanu, "Problèmes globaux dans le théorie des équations intégrales de Volterra", Ann. Mat. Pura Appl. 67 (1965), 349-363. 
[5] Robert M. Fortet, "Random distributions with an application to telephone engineering", Proc. Third Berkeley Sympos. Math. Statist. Probability, 2 (1954-1955), 81-88 (University of California Press, Berkeley, Los Angeles, 1956).

[6] J.L. Kelley, General topology (Van Nostrand, Toronto, New York, London, 1955).

[7] Michel Loève, Probability theory, 3rd ed. (Van Nostrand, Princeton, New Jersey; Toronto, Ontario; London; 1963).

[8] J. Susan Milton, W.J. Padgett and Chris P. Tsokos, "On the existence and uniqueness of a random solution to a perturbed random integral equation of the Fredholm type", SIAM J. Appl. Math. 22 (1972), 194-208.

[9] T. Morozan, "Stability of linear systems with random parameters", $J$. Differential Equations 3 (1967), 170-178.

[10] M.Z. Nashed and J.S.W. Wong, "Some variants of a fixed point theorem of Krasnoselskii and applications to nonlinear integral equations", J. Math. Mech. 18 (1969); 767-777.

[11] W.J. Padgett and C.P. Tsokos, "On a semi-stochastic model arising in a biological system", Math. Biosci. 9 (1970), 105-117.

[12] W.J. Padgett and C.P. Tsokos, "A stochastic model of chemotheraphy: Computer similation", Math. Biosci. 9 (1970), 119-133.

[13] W.J. Padgett and C.P. Tsokos, "On a stochastic integral equation of the Volterra type in telephone traffic theory", J. App $Z$. Probability 8 (1971), 269-275.

[14] W.J. Padgett and C.P. Tsokos, "Existence of a solution of a stochastic integral equation in turbulence theory", J. Math. Phys. 12 (1971), 210-212.

[15] C.P. Tsokos, "On some stochastic differential systems", Proc. Third Annual Princeton Conf. on Inf. Sciences and Systems, 1969, 228-234.

[16] Chris P. Tsokos, "On the classical stability theorem of PoincaréLyapunov with a random parameter", Proc. Jopan Acad. 45 (1969), $781-785$. 
[17] Chris P. Tsokos, "On a stochastic integral equation of the Volterra type", Math. Systems Theory 3 (1969), 222-23l.

[18] C.P. Tsokos and M.A. Hamdan, "Stochastic nonlinear integrodifferential systems with time-lag", J. Natur. Sci. and Math. 10 (1970), 293-303.

[19] Chris P. Tsokos and M.A. Hamdan, "Stochastic asymptotic exponential stability of stochastic integral equations", J. Appl. Probability 9 (1972), 169-177.

Department of Mathematics,

University of South Carolina,

Col umb i a,

South Carolina,

USA. 